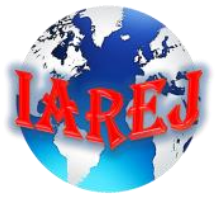

Research Article

\title{
Analysis and modified function projective synchronization of integer and fractional-order autonomous Morse jerk oscillator
}

\author{
Eric Donald Dongmo $^{a}$ (D), Cyrille Ainamon ${ }^{b}$ (D), Alex Stephane Kemnang Tsafack ${ }^{c, *}$ (D), \\ Nasr Saeed ${ }^{d}$ (D), Victor Kamdoum Tamba ${ }^{e}$ (D) and Sifeu Takougang Kingni ${ }^{f}$ (D) \\ ${ }^{a}$ Department of Mechanical Engineering, College of Technology, University of Buea, P. O. Box: 63 Buea, Cameroon \\ ${ }^{b}$ Institute of Mathematics and Physical Sciences, University of Abomey-Calavi, P. O. Box: 613, Porto Novo, Benin \\ ${ }^{c}$ Research unit of Condensed Matter of Electronics and Signal Processing, Department of Physics, Faculty of Sciences, University \\ of Dschang, P.O. Box: 67, Dschang, Cameroon \\ ${ }^{d}$ Department of Physics, College of Education, Nyala University, P.O. Box: 155, Nyala, Sudan \\ ${ }^{e}$ Department of Telecommunication and Network Engineering, IUT-Fotso Victor of Bandjoun, University of Dschang, P. O. Box: \\ 134, Bandjoun, Cameroon \\ ${ }^{f}$ Department of Mechanical, Petroleum and Gas Engineering, Faculty of Mines and Petroleum Industries, University of Maroua, \\ P.O. Box: 46, Maroua, Cameroon
}

\begin{tabular}{l}
\hline ARTICLE INFO \\
\hline Article history: \\
Received 07 January 2021 \\
Revised 29 April 2021 \\
Accepted 21 May 2021 \\
\hline Keywords: \\
Chaos \\
Electronic implementation \\
Integer and fractional-order \\
Modified function projective \\
Morse jerk oscillator \\
Periodic spiking oscillations \\
Synchronization
\end{tabular}

\begin{abstract}
Dynamical analysis and modified function projective synchronization (MFPS) of integer and fractional-order Morse jerk oscillator are investigated in this paper. Integerorder Morse jerk oscillator generates periodic behaviors, periodic spiking and two different shapes of chaotic attractors. The periodic spiking and chaotic behaviors obtained during numerical simulations of integer-order Morse jerk oscillator is ascertained by using electronic implementation. The numerical simulations results qualitatively agree with the Orcad-PSpice results. Moreover, MFPS of identical and mismatched chaotic Morse jerk oscillators is numerically investigated. At last, the theoretical investigation of fractional-order Morse jerk oscillator reveals the existence of chaos in Morse jerk oscillator for order greater or equal to 2.85 .
\end{abstract}

(C) 2021, Advanced Researches and Engineering Journal (IAREJ) and the Author(s).

\section{Introduction}

Chaotic systems are highly sensitive to petty perturbations [1]. Chaos can be observed in various physical systems [2-5] and it is useful in many fields [6, 7]. The construction of chaotic oscillators with simple structures [8-14] is an interesting field of research. There is two types of simple chaotic oscillators: Nonautonomous [9] and autonomous oscillators [10-14]. Sprott in [14] proposed various new chaotic jerk oscillators with diverse nonlinearities and easy electronic implementation.

Following the idea of proposing new autonomous chaotic oscillators with easy electronic implementation, several research works have been conducted on new autonomous chaotic jerk oscillators [12-17]. The authors of [15-17] introduced autonomous chaotic jerk oscillators using Duffing and Van der Pol dynamics washed into a jerk system. The authors of [15, 16] proposed an autonomous chaotic Van der Pol jerk oscillator based on second-order-equation of Van der Pol oscillator. The authors of [17] proposed an autonomous chaotic Duffing jerk oscillator based on second-order-equation of Duffing oscillator. Inspired by [12-17], Morse jerk oscillator is designed based on the second-order autonomous Morse oscillator in this paper. Then dynamical analysis, synchronization, circuit design of integer and fractional-

* Corresponding author. Tel.: +237-698-022-585.

E-mail addresses: eric.dongmo90@gmail.com (E.D.Dongmo), ainamoncyrille@yahoo.fr (C.Ainamon), alexstephanekemnang@gmail.com (A.S.K.Tsafack), nasrsaeed19@yahoo.com (N. Saeed), v.kamdoum@gmail.com (V.K.Tamba), stkingni@gmail.com ( S.T. Kingni) ORCID: 0000-0002-0203-2829 (E.D. Dongmo), 0000-0002-3656-1614 (C. Ainamon), 0000-0002-4602-9293 (A.S.K. Tsafack), 0000-0002-5123-5139 (N. Saeed), 0000-0002-0835-4722 (V.K. Tamba), 0000-0003-1547-6856 (S.T. Kingni) DOI: 10.35860 /iarej.854623

This article is licensed under the CC BY-NC 4.0International License (https://creativecommons.org/licenses/by-nc/4.0/). 
order Morse jerk oscillator are studied. The fractionalorder of autonomous Morse jerk oscillator is analysed by using analytical [18] and numerical methods [19, 20]. Morse oscillator is used to describe molecular vibrations $[21,22]$. The present work contributes to the dynamical analysis, the modified function projective synchronization (MFPS) of integer and fractional-order Morse jerk oscillator. Integer-order of this jerk oscillator generates periodic behaviors, periodic spiking and chaotic attractors. Investigations of fractional-order Morse jerk oscillator reveals the existence of chaos in Morse jerk oscillator for order less than three.

This paper is organized as follows: In the Section 2, the Morse jerk oscillator is analysed and implemented on Orcad-PSpice software. The MFPS of identical and mismatched coupled chaotic Morse jerk oscillators is investigated in Section 3. Section 4 deals with the analysis of fractional-order Morse jerk oscillator. Section 5 presents the conclusion.

\section{Dynamical Analysis of Morse Jerk Oscillator}

The two-dimensional Morse equation [22] can be transformed to a jerk oscillator:

$$
\begin{gathered}
\dot{x}=y, \\
\dot{y}=\delta z, \\
\dot{z}=-\gamma y-z-\beta[1-\exp (-\alpha x)] \exp (-\alpha x)
\end{gathered}
$$

where $\alpha, \gamma, \beta, \delta$ are positive parameters, $\ddot{x}(t)=$ $z(t)$ and $\dot{x}(t)=y(t)$. For $\delta=\alpha=1, \gamma=0.16$ and by increasing the parameter $\beta$, system (2) exhibits steady sate behaviour up to $\gamma=\beta=0.16$ where a Hopf bifurcation appears followed by limit cycle and periodic spiking oscillations as illustrated in Figure 1.
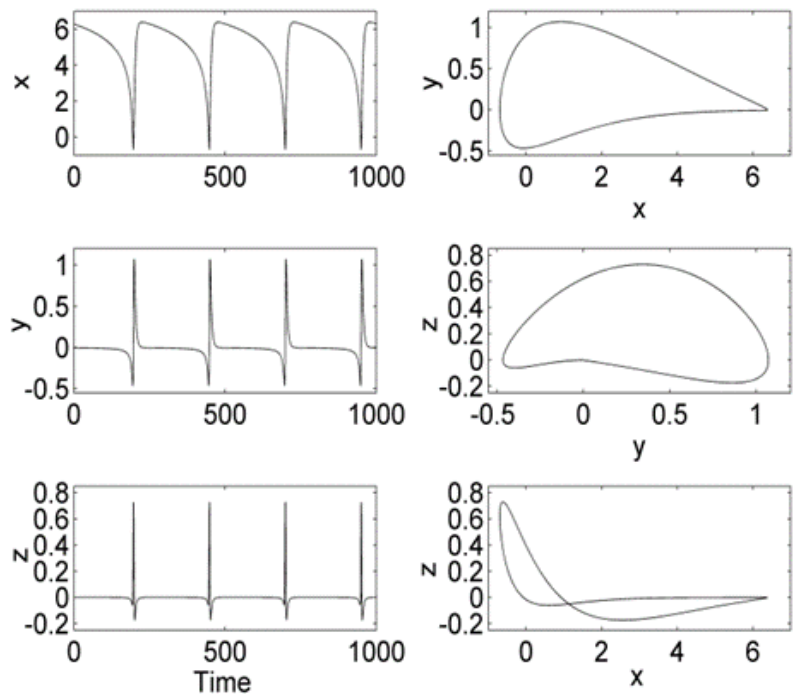

Figure 1. Time traces (left panel) and phase portraits (right panel) for $\alpha=1, \gamma=0.16$ and $\beta=0.5$ and the initial conditions $(\boldsymbol{x}(\mathbf{0}), \boldsymbol{y}(\mathbf{0}), \boldsymbol{z}(\mathbf{0}))=(0.1,0.1,0.1)$
The periodic spiking oscillations aredepicted in Figure 1. System (1) is dissipative and has single equilibrium point $O(0,0,0)$. The characteristic equation at the equilibrium point $O=(0,0,0)$ is:

$$
\lambda^{3}+\lambda^{2}+\gamma \delta \lambda+\alpha \beta \delta=0
$$

Based on Routh-Hurwitz criteria, the single equilibrium point is stable if $\gamma>\alpha \beta$ and unstable for $\gamma<$ $\alpha \beta$ because $\delta>0$.

Figure 2 presents the two parameters bifurcation diagram of system (1) for $\alpha=1$ and $\gamma=0.16$.

System (1) exhibits periodic (grey color) and chaotic (black color) behaviors as depicted in Figure 2. Figure 3 presents the phase portraits of chaotic behaviors for given values of $\beta$ and $\delta$.

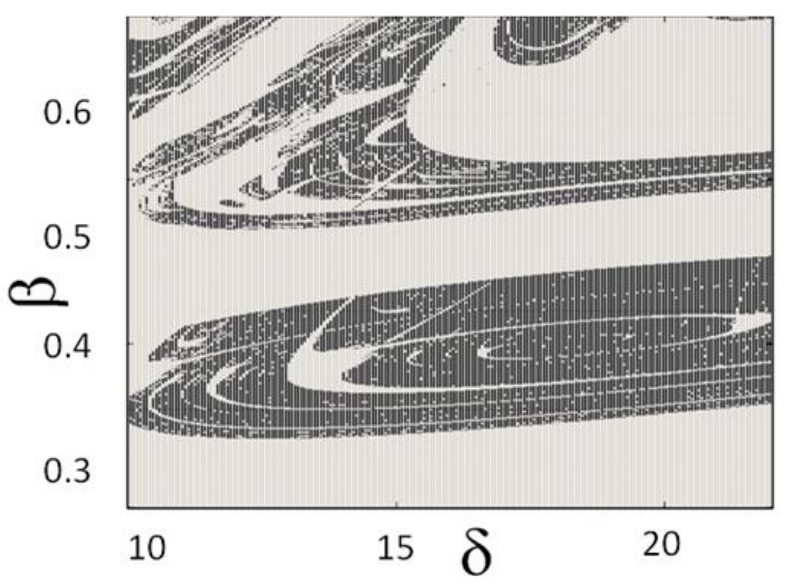

Figure 2. Two parameters $(\delta, \beta)$ bifurcation diagram for $\alpha=$ 1 and $\gamma=0.16$.
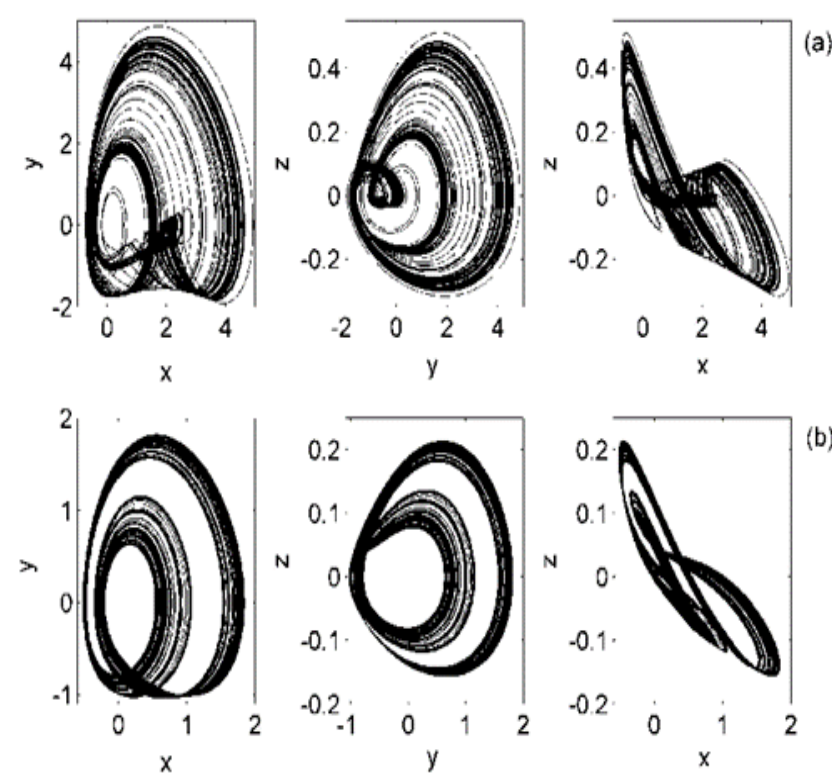

Figure 3. Phase portraits of system (1) for given values of $\delta$ and $\beta$ :(a) $\beta=0.6, \delta=18$ and (b) $\beta=0.345, \delta=12.85$.

The others parameters are $\alpha=1$ and $\gamma=0.16$. The initial conditions are $(\boldsymbol{x}(\mathbf{0}), \boldsymbol{y}(\mathbf{0}), \boldsymbol{z}(\mathbf{0}))=(0.1,0.1,0.1)$ 
From Figure 3, it emerges two different forms of chaotic attractors for given values of $\beta$ and $\delta$. The circuit of system (1) is depicted in Figure 4. This figure includes of analog multiplier device (ADJ633), operational amplifiers (TL084), capacitors, resistors and battery. The exponential black box is built by an operational amplifier associated with a resistor and a single diode. The phase portraits obtained from the Orcard-PSpice software are shown in Figure 5.

The obtained PSpice results of Figure 5 are in agreement with the numerical results of the right panel of Figure 1 and Figure 3.

\section{Modified Function Projective Synchronization of Chaotic Morse Jerk Oscillators}

To achieve the MFPS [23, 24] of unidirectional coupled chaotic Morse jerk oscillators, the controllers are designed for MFPS of identical and mismatched Morse jerk oscillators. The numerical simulations are included in each case to ascertain the effectiveness of the control method. The chaotic drive and response systems are described by Equations (3) and (4), respectively.

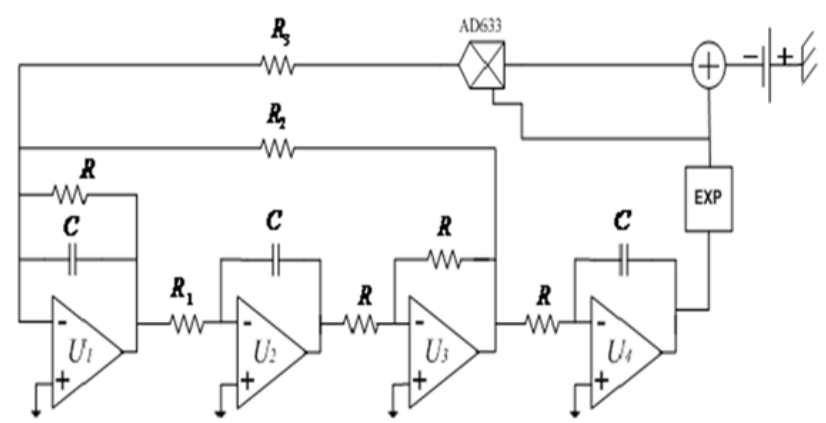

Figure 4. Circuit illustrating system (1)

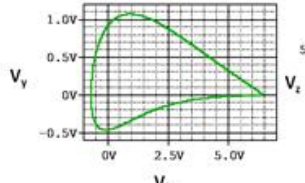

$v_{x}$

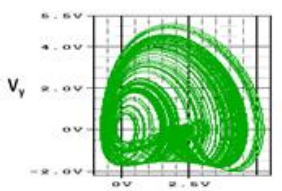

$v_{x}$
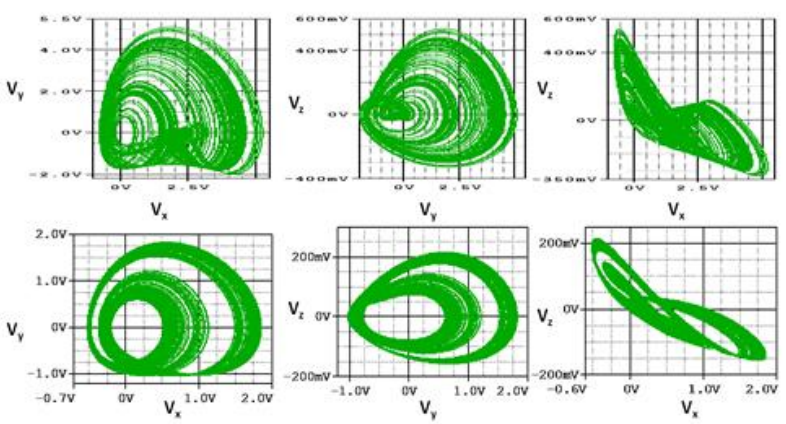

$v_{\mathrm{y}}$

Figure 5. Phase portraits of periodic and chaotic attractors obtained on Orcard-PSPICE. Resistors and capacitor values are $C=10 \mathrm{nF}, R_{1}=R=10 \mathrm{k} \Omega, R_{3}=20 \mathrm{k} \Omega, R_{2}=62.50 \mathrm{k} \Omega$

(first line reproduces the right panel of Figure 1), $R_{3}=$ $16.16 \mathrm{k} \Omega, R=10 \mathrm{k} \Omega, R_{1}=555.5 \mathrm{k} \Omega, R_{2}=62.5 \mathrm{k} \Omega, C=10 \mathrm{nF}$ (second line agrees with Figure $3(\mathrm{a})$ ) and $R=10 \mathrm{k} \Omega, R_{1}=$ $778.21 \mathrm{k} \Omega, R_{2}=62.5 \mathrm{k} \Omega, R_{3}=28.98 \mathrm{k} \Omega$ (third line agrees with Figures 3(b)).

$$
\begin{gathered}
\dot{x}=f(x)+\Delta f(x) \\
\dot{y}=f(y)+D(y, \tilde{g})
\end{gathered}
$$

where $x, y \in R^{n}$ are the state vectors, $f$ is the differentiable vector function, $\Delta f(x)$ is the mismatched term and equal to zero for identical oscillators. The coupling function $D(y, \tilde{g})$ is given by Equation (5).

$$
\begin{aligned}
D(y, \tilde{g})=\Lambda_{i} m(t) \dot{x}(t) & +\Lambda_{i} \dot{m}(t) x(t)-f(\tilde{g}) \\
& +(H-J F(\tilde{g}(t)))(y \\
& \left.-\Lambda_{i} m(t) x(t)\right)
\end{aligned}
$$

where the constant diagonal matrix is $\Lambda_{i}=\operatorname{diag}\left(\Lambda_{1}\right.$, $\left.\Lambda_{2}, \ldots, \Lambda_{n}\right), m(t)$ is a differentiable function with $m(t) \neq$ 0 for all $\mathrm{t}, \tilde{g}(t)$ is the goal dynamics function which is defined by Equation (6).

$$
\begin{gathered}
\tilde{g}(t)=\left[g_{1}(t), g_{2}(t), \ldots, g_{n}(t)\right]^{T} \\
=\left[\Lambda_{1} m(t) x_{1}(t), \Lambda_{2} m(t) x_{2}(t), \ldots, \Lambda_{n} m(t) x_{n}(t)\right]^{T}
\end{gathered}
$$

where $H$ is an arbitrary constant Hurwitz matrix $(n \times n)$ whose eigenvalues all have negative real parts and $\frac{J=\partial}{\partial \tilde{g}}$ is the Jacobian matrix of the dynamical system. The error state vector of MFPS is given by Equation (7).

$$
e(t)=y(t)-\tilde{g}(t)
$$

The challenge is to build the response jerk oscillator in order its synchronize with the drive jerk oscillator for given scaling diagonal matrix and $\mathrm{m}(\mathrm{t})$ :

$$
\lim _{t \rightarrow \infty}\left\|y(t)-\Lambda_{i} m(t)(t) x(t)\right\|=0
$$

\subsection{MFPS of Unidirectional Coupled Identical Chaotic Morse Jerk Oscillators}

The drive chaotic Morse jerk oscillator is described by Equation (9).

$$
\begin{gathered}
\dot{x}_{1}=x_{2}, \\
\dot{x}_{2}=\delta x_{3},
\end{gathered}
$$$$
\dot{x}_{3}=-\gamma x_{2}-x_{3}-\beta\left[1-\exp \left(-\alpha x_{1}\right)\right] \exp \left(-\alpha x_{1}\right) \text {. }
$$

By applying the OPCL coupling, the response oscillator is given by Equation (10).

$$
\begin{gathered}
\dot{y}_{1}=y_{2}+m(t)\left(\Lambda_{1}-\Lambda_{2}\right) x_{2}+\Lambda_{1} \dot{m}(t) x_{1}-e_{1}-e_{2}, \\
\dot{y}_{2}=\delta y_{3}+m(t)\left(\Lambda_{2}-\Lambda_{3}\right) \delta x_{3}+\Lambda_{2} \dot{m}(t) x_{2}-e_{2} \\
-\delta e_{3}, \\
\dot{y}_{3}=-y_{3}-\gamma y_{2}-\beta\left[1-\exp \left(-\alpha y_{1}\right)\right] \exp \left(-\alpha y_{1}\right) \\
+m(t)\left(\Lambda_{2}-\Lambda_{3}\right) \gamma x_{2}+\Lambda_{3} \dot{m}(t) x_{3} \\
\Lambda_{3} m(t) \beta\left[1-\exp \left(-\alpha x_{1}\right)\right] \exp \left(-\alpha x_{1}\right) \\
+\beta\left[1-\exp \left(-\alpha \Lambda_{1} m(t) x_{1}\right)\right] \exp \left(-\alpha \Lambda_{1} m(t) x_{1}\right) \\
-b e_{1}+\gamma e_{2} .
\end{gathered}
$$


where $a=\alpha \beta\left[1-2 \exp \left(-\alpha x_{1}\right)\right] \exp \left(-\alpha x_{1}\right)$ and $b=$ $\alpha \beta\left[1-2 \exp \left(-\alpha \Lambda_{1} m(t) x_{1}\right)\right] \exp \left(-\alpha \Lambda_{1} m(t) x_{1}\right)$. The parameter values using during numerical simulations of the drive and response jerk oscillator are those of Figure 3 (a) for which the oscillator presents a chaotic behavior. The constant diagonal matrix is taken arbitrary as $\Lambda=$ $\operatorname{diag}(0.1,0.2,0.3)$ whereas the differentiable function is $m(t)=3+1.5 \sin (2 \pi t / 10)$. The numerical simulations results are presented in Figure 6. Figure 6 (a) presents the time traces of the state vector $x_{1}$ (solid line for the drive jerk) and $y_{1}$ (dashed line the response jerk oscillator) respectively for the drive and the response oscillators. Figure 6 (b) shows the time traces of MFPS errors $e_{i}(t) i=1,2,3$ tending to zero asymptotically showing the achievement of MFPS between drive and response systems. Figure 6 (c) shows that $\frac{\|y\|}{\|x\|}$ tends to the differentiable function $m(t)$ indicating MFPS.

\subsection{MFPS of Two Mismatched Chaotic Morse Jerk \\ Oscillators}

For $\alpha=1$, the mismatched chaotic Morse jerk oscillator is defined by Equation (11).

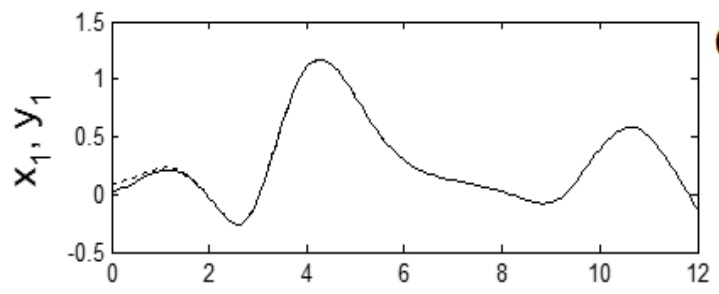

(a)

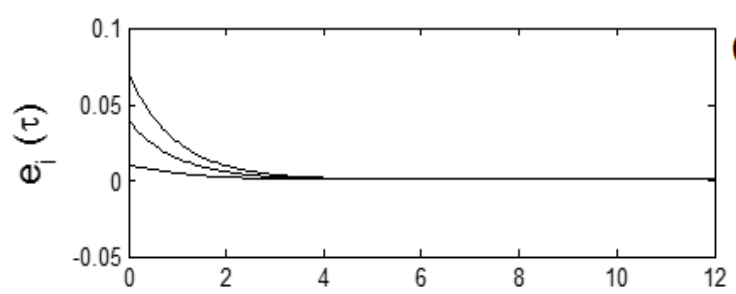

(b)

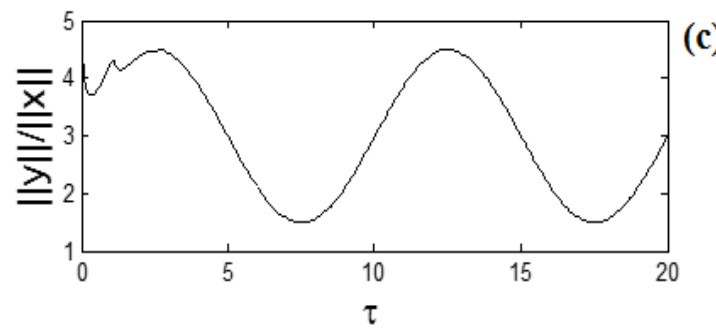

Figure 6. MFPS of identical chaotic Morse jerk oscillators: (a) Time traces of the state vector $x_{1}$ (solid line) and $y_{1}$ (dashed line), (b) evolution errors and

$$
\begin{gathered}
\text { (c) ratio } \frac{\|y\|}{\|x\|} \text {. The initial conditions are } \\
\qquad\left(y_{1}(0), y_{2}(0), y_{3}(0)\right)=(0.1,0.1,0.1) \\
\text { and }\left(x_{1}(0), x_{2}(0), x_{3}(0)\right)=(0.1,0.1,0.1) .
\end{gathered}
$$

$$
\begin{gathered}
\dot{x}_{1}=x_{2} \\
\dot{x}_{2}=\delta x_{3}+\Delta \delta x_{3} \\
\dot{x}_{3}=-x_{3}-\gamma x_{2}-\beta\left[1-\exp \left(-x_{1}\right)\right] \exp \left(-x_{1}\right) \\
-\Delta \gamma x_{2} \\
-\Delta \beta\left[1-\exp \left(-x_{1}\right)\right] \exp \left(-x_{1}\right) .
\end{gathered}
$$

System (11) is still chaotic for $\beta=0.6, \Delta \beta=0.3, \gamma=$ $0.16, \Delta \gamma=0.1, \delta=18$ and $\Delta \delta=3$. Considering (11) as the drive jerk, the response jerk is given by Equation 12 .

$$
\begin{aligned}
& \dot{y}_{1}=y_{2}+m(t)\left(\Lambda_{1}-\Lambda_{2}\right) x_{2}+\Lambda_{1} \dot{m}(t) x_{1}-e_{1}-e_{2} \text {, } \\
& \dot{y}_{2}=\delta y_{3}+\Delta \delta y_{3}+m(t)\left(\Lambda_{2}-\Lambda_{3}\right) \delta x_{3}+ \\
& m(t)\left(\Lambda_{2}-\Lambda_{3}\right) \Delta \delta x_{3}+\Lambda_{2} \dot{m}(t) x_{2}-e_{2}-\delta e_{3} \text {, } \\
& \dot{y}_{3}=-y_{3}-\gamma y_{2}-\beta \exp \left(-y_{1}\right)\left[1-\exp \left(-y_{1}\right)\right] \\
& -\Delta \gamma y_{2} \\
& -\Delta \beta \exp \left(-y_{1}\right)\left[1-\exp \left(-y_{1}\right)\right] \\
& +m(t)\left(\Lambda_{2}-\Lambda_{3}\right) \gamma x_{2}+\Lambda_{3} \dot{m}(t) x_{3} \\
& -\Lambda_{3} m(t)(\beta+\Delta \beta)[1 \\
& \left.-\exp \left(-x_{1}\right)\right] \exp \left(-x_{1}\right) \\
& +m(t)\left(\Lambda_{2}-\Lambda_{3}\right) \Delta \gamma x_{2} \\
& +(\beta+\Delta \beta)[1 \\
& \left.-\exp \left(-\Lambda_{1} m(t) x_{1}\right)\right] \exp \left(-\Lambda_{1} m(t) x_{1}\right) \\
& -c e_{1}+\gamma e_{2}, \\
& c=\beta\left[1-2 \exp \left(-\Lambda_{1} m(t) x_{1}\right)\right] \exp \left(-\Lambda_{1} m(t) x_{1}\right) \text {. }
\end{aligned}
$$

The parameter values using during numerical simulations of the drive and response jerks are those of Figure 3 (a). The mismatch parameters are chosen as $\Delta \delta=1, \Delta \gamma=0.1$ and $\Delta \beta=0.2$. The constant diagonal matrix is taken arbitrary as $\Lambda=\operatorname{diag}(0.3,0.2,0.1)$ while the differentiable function as $m(t)=5+$ $2 \sin (2 \pi t / 10)$. Sample numerical simulations results are presented in Figure 7.

Figure 7 (a) presents the time traces of the state vector $x_{1}$ (solid line) and $y_{1}$ (dashed line). Figure 7 (b) shows that the time traces of MFPS errors $e_{i}(t) i=1,2,3$ tend to zero asymptotically indicating MFPS between drive and response oscillators in presence of mismatch parameters. Figure 7 (c) shows that $\frac{\|y\|}{\|x\|}$ tends to $m(t)$ confirming MFPS.

\section{Fractional-Order of Chaotic Morse Jerk Oscillator}

The rate-equations describing of the fractional order of

$$
\begin{gathered}
\frac{d^{q_{1}} x}{d t^{q_{1}}}=y, \\
\frac{d^{q_{2} y}}{d t^{q_{2}}}=\delta z,
\end{gathered}
$$

$\frac{d^{q_{3}} z}{d t^{q_{3}}}=-\gamma y-z-\beta \exp (-\alpha x)[1-\exp (-\alpha x)]$.

system (1) is given by Equation (13). 

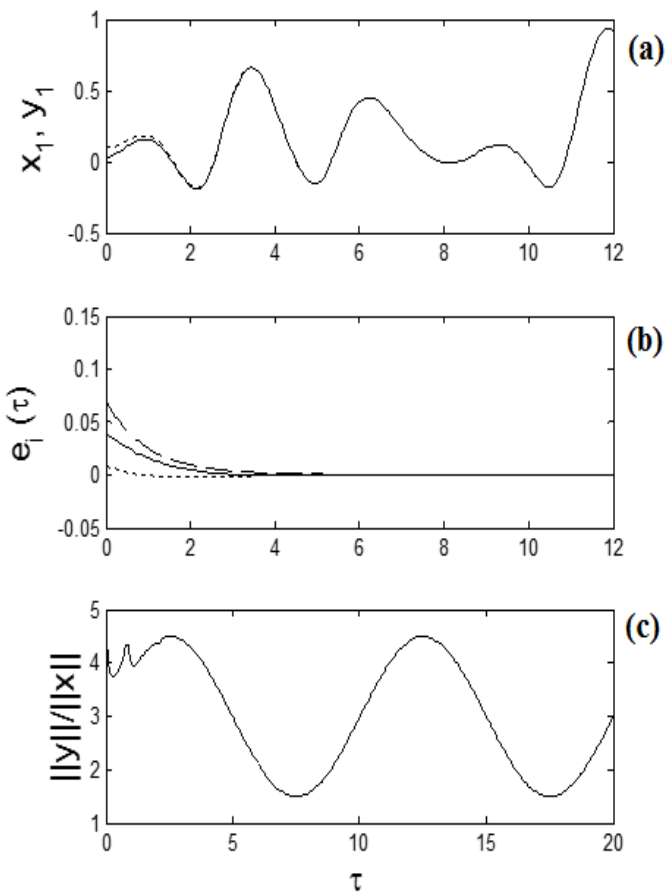

Figure 7. MFPS of mismatched chaotic Morse jerk oscillators.

(a) Time traces of the state vector $x_{1}$ (solid line) and $y_{1}$ (dashed line) and in presence of mismatch parameters, (b) evolution errors and (c) ratio $\frac{\|y\|}{\|x\|}$. The initial conditions are

$$
\left(x_{1}(0), x_{2}(0), x_{3}(0)\right)=
$$

$(0.1,0.1,0.1)$ and $\left(y_{1}(0), y_{2}(0), y_{3}(0)\right)=(0.1,0.1,0.1)$
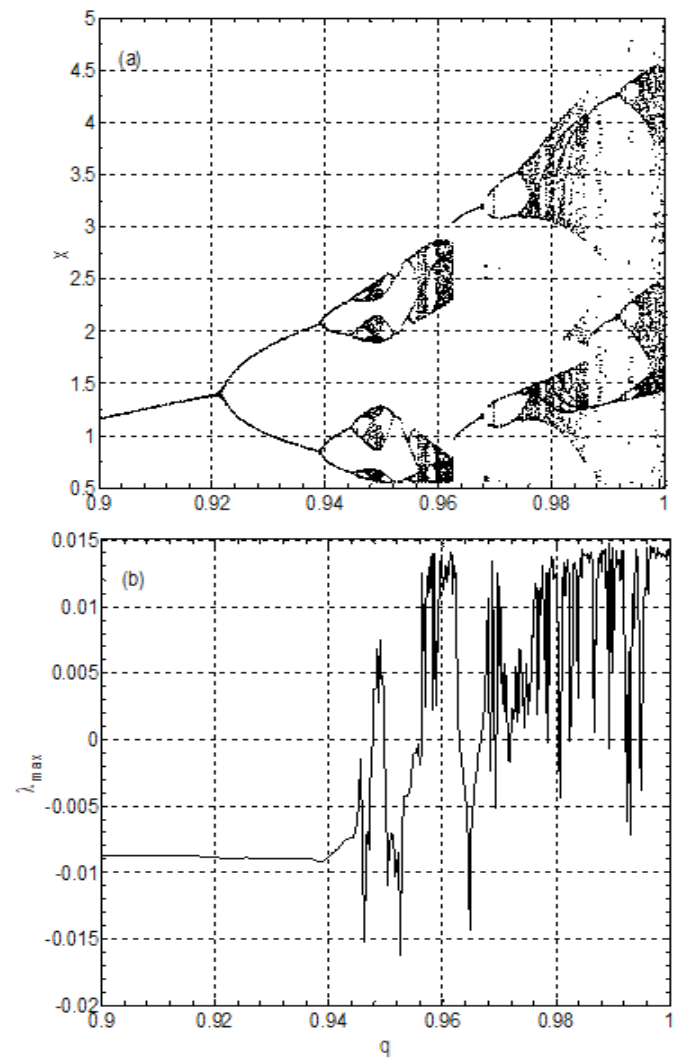

Figure 8. Bifurcation diagram of $x$ (a) and LLE (b) of system (13) as function of the parameterqwith $\alpha=1, \gamma=0.16, \delta=$ 18 and $\beta=0.6$.
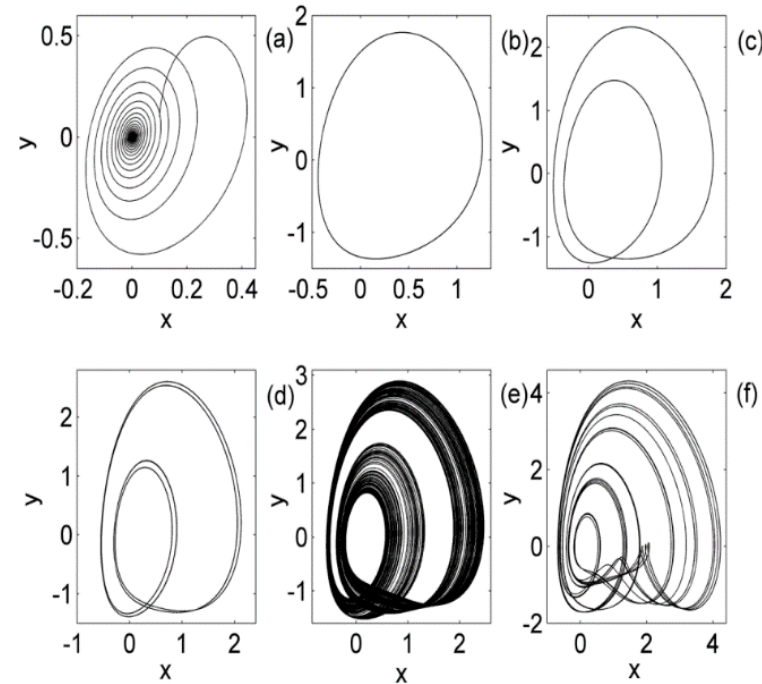

Figure 9. Phase portraits of fractional order system (13) for specific values of parameterq: (a) $q=0.83$, (b) $q=0.91$, (c) $q=0.93$, (d) $q=0.941$, (e) $q=0.95$ and (f) $q=0.99$.

where $\quad q_{1}=q_{2}=q_{3}=q(0<q<1) \quad$ is the commensurate fractional order. For $\alpha=1, \gamma=0.16$, $\delta=18$ and $\beta=0.6$, the eigenvalues of equilibrium point $O(0,0,0)$ are given as $\lambda_{1}=-2.091656729,: \lambda_{2,3}=$ $0.5458283647 \pm 2.205774816 j$. Based on Eq. (17) in [21], the fractional-order system (13) exhibits chaos if $3 q_{0} \geq 2.536703964$. The bifurcation diagram representing the local maxima of $x(t)$ and LLE versus of parameter $q$ are plotted in Figure 8 .

In Figure 8, system (13) exhibits limit cycle and period-doubling route to chaos incrusted with periodic windows. So, system (13) exhibits chaos for $3 q_{0} \approx 2.85$. Figure 9 depicts the phase portraits of system (13) for given value of parameterq.

Figure 9 exhibits steady state behavior at $q=0.83$ (see Figure 9 (a)), limit cycle at $q=0.941$ (see Figure 9 (b)), period doubling attractors (see Figures 9 (c) and (d)) to chaotic attractors (see Figures 9 (e) and (f)).

\section{Conclusions}

This paper investigated the dynamics and modified function projective synchronization of integer and fractional-order Morse jerk oscillator. Interesting dynamics such as periodic oscillations, periodic spiking and two different shapes of chaotic attractors emerged by tuning the parameters. The designed circuit of autonomous Morse jerk oscillator was realized on the Orcad-PSpice software to ascertain the numerical simulations results. Next, numerical simulations of identical and mismatched chaotic Morse jerk oscillators were done to denote the effectiveness of the modified function projective synchronization. At last, the theoretical investigation of fractional-order Morse jerk 
oscillator revealed the existence of chaos in Morse jerk oscillator for order greater or equal to 2.85 .

\section{Declaration}

The authors declared no potential conflicts of interest with respect to the research, authorship, and/or publication of this article. The authors also declared that this article is original, was prepared in accordance with international publication and research ethics, and ethical committee permission or any special permission is not required.

\section{Author Contributions}

N. Saeed, V.K. Tamba and S.T. Kingni conceived this study. E.D. Dongmo, C. Ainamon and A.S.K. Tsafack did the calculations and computers simulations. E. D. Dongmo and A.S.K. Tsafack did the analogsimulations. E.D. Dongmo, C. Ainamon and A.S.K. Tsafack wrote the manuscript. N. Saeed, V.K. Tamba and S.T. Kingni checked the results obtained in this study.

\section{References}

1. Ivancevic V.G. and T.T. Ivancevic, Complex nonlinearity: chaos, phase transitions, topology change, and path integrals. 2008, Berlin: Springer Science \& Business Media.

2. Rial J.A., Abrupt climate change: chaos and order at orbital and millennial scales. Global and planetary change, 2004. 41: p. 95-109.

3. Carlen E., R. Chatelin, P. Degond and B. Wennberg, Kinetic hirerachy and propagation of chaos in biological swarm models. Physica D: Nonlinear Phenomena, 2013. 260: p. 90-111.

4. Callan K. L., L. Illing, Z. Gao, D. J. Gauthier and E. Schöll, Broadband chaos generated by an optoelectronic oscillator. Physical Review Letters, 2010. 104: 113901.

5. Kingni S. T., G. Van der Sande, I. V. Ermakov and J. Danckaert, Theoretical analysis of semiconductor ring laser with short and long time-delayed optoelectronic and incoherent feedback. Optics Communications, 2015. 341: p. 147-154.

6. Nana B., P. Woafo and S. Domngang, Chaotic synchronization with experimental application to secure communications. Communications in nonlinear science and Numerical Simulation, 2009. 14: p. 2266-2276.

7. Kingni S. T., J. H. TallaMbé and P. Woafo, Semiconductor lasers driven by self-sustained chaotic electronic oscillators and applications to optical chaos cryptography. Chaos, 2012. 22: p. 033108-033115.

8. Sprott J. C., Elegant Chaos Algebraically Simple Flows. 2010, Singapore: World Scientific.

9. Lindberg E., K. Murali and A. Tamasevicius, The smallest transistor-based nonautonomous chaotic circuit. IEEE Transactions on Circuits and Systems II: Express Briefs, 2005. 52: p. 661-664.

10. Tchitnga R., H. B. Fotsin, B. Nana, P. H. L. Fotso and P. Woafo, Hartley's oscillator: the simplest chaotic twocomponent circuit. Chaos Solitons Fractals, 2012. 45: p. 306-313.
11. Muthuswamy B. and L. O. Chua, Simplest chaotic circuit. International Journal of Bifurcation and Chaos, 2010. 20: p. $1567-1580$.

12. Piper J. R. and J. C. Sprott, Simple autonomous chaotic circuits, IEEE Transactions on Circuits and Systems II: Express Briefs, 2010. 57: p. 730-734.

13. Srisuchinwong B. and B. Munmuangsaen, Current-tunable chaotic jerk oscillator. Electronics letters, 2012. 48: p. 1051-1053.

14. Sprott J.C., Simple chaotic systems and circuits. American Journal of Physics, 2000. 68: p. 758-763.

15. Acho L., J. Rolon and S. Benitez, Synchronization of mechanical systems with a new Van der Pol chaotic oscillator. WSEAS Transactions on Circuits and Systems, 2004. 3: p. 198-199.

16. Benitez S., L. Acho and R. J. R. Guerra, Chaotification of the Van der Pol system using Jerk architecture. IEICE Transactions on Fundamentals of Electronics, Communications and Computer Sciences, 2006. 89: p. 1088-1091.

17. Kengne J., Z. T. Njitacke and H. B. Fotsin, Dynamical analysis of a simple autonomous jerk system with multiple attractors, Nonlinear Dynamics, 2016. 83(1): p. 751-765.

18. Tavazoei M. S., M. Haeri, A necessary condition for double scroll attractor existence in fractional-order systems. Physics Letters A, 2007. 367(1-2): p.102-13.

19. Caponetto R., R. Dongola, L. Fortuna and I. Petraš, Fractional-order system: Modelling and control applications. 2010, World scientific series on nonlinear science, series A, vol. 72 .

20. Diethelm K., N. J. Ford, D. Freed, A predictor-corrector approach for the numerical solution of fractional differential equations. Nonlinear Dynamics 2002. 29(1): p. $3-22$.

21. $\mathrm{Wu}$ J. and J. Cao, Linear and nonlinear response functions of the Morse oscillator: Classical divergence and the uncertainty principle, The Journal of Chemical Physics, 2001. 115(12): p. 5381-5392.

22. Jing Z., J. Deng and J. Yang, Bifurcations of periodic orbits and chaos in damped and driven Morse oscillator. Chaos, Solitons \& Fractals, 2008. 35(3): p. 486-505.

23. Sudheer K.S. and M. Sabir, Modified function projective synchronization of hyperchaotic systems through openplus-closed-loop coupling. Physics Letters A, 2010. 374(19-20): p. 2017-2023.

24. Kamdoum Tamba V., H. B. Fotsin, J. Kengne, F Kapche Tagne and P. K. Talla, Coupled inductors-based chaotic Colpitts oscillators: Mathematical modeling and synchronization issues. The European Physical Journal Plus, 2015. 130(7):137. 